121 THE INTRODUCTION OF AN OPIOID PATCH MONITORING CHART ONTO INPATIENT ONCOLOGY WARDS AT ST BARTHOLOMEW'S HOSPITAL

Anna Bradley, David Feuer. Barts Health NHS Trust

10.1136/bmjspcare-2019-ASP.144

Background The urgent need to improve patient safety with regards to opioid prescribing on the specialist oncology inpatient wards at St Bartholomew's Hospital was highlighted in an audit conducted by the hospital palliative care team in 2017. This demonstrated multiple opioid prescribing and administration errors, including for transdermal patches. The introduction of an opioid patch monitoring chart, as well as a robust education programme for all clinical staff, was recommended.

Errors relating to transdermal patches often include: new patches being applied prior to old ones being removed, failure to recognise patches falling off/becoming displaced and multiple different opioid patches being applied in different locations. Through regular documented nursing checks on an opioid patch monitoring chart it would be hoped that these errors could be avoided, as well as improving knowledge and competency around opioid prescribing.

Methods A consultation was held amongst the palliative care and oncology teams regarding the introduction of the chart. An existing chart was adapted with appropriate permissions. The chart was implemented in June 2018 with extensive teaching sessions provided.

Results A prospective audit of the chart's use was conducted and feedback obtained from users. The results demonstrated that the chart was well used. Only one patient prescribed an opioid patch did not have the appropriate chart in their notes and in only one circumstance was the chart completed incorrectly.

$81.8 \%$ of surveyed staff found the chart 'very easy' to use and $100 \%$ felt that the chart improved patient safety.

Conclusions The opioid patch monitoring chart has been well imbedded into the oncology wards. It is now planned to introduce it across the rest of Barts Health NHS Trust.

\section{COMMUNICATION OF ADVANCE CARE PLANNING DECISIONS}

James Artingstall, Helen Pegrum, Sophie Strang. Florence Nightingale Hospice

10.1136/bmjspcare-2019-ASP.145

Background The creation of an Advance Care Plan (ACP) with patients under the care of a specialist palliative medicine team is a crucial part of their role. It is important that such discussions are documented effectively, to accurately reflect the patient's wishes and protect their autonomy. The appropriate dissemination of an ACP to key professionals involved in the care of such patients is equally important; to ensure continuity of care across the multidisciplinary team.

Methods We set out to assess the prevalence and subsequent communication of clearly documented ACP's in the electronic and Palliative Care-held notes in patients admitted to a hospice for any reason. Through analysis of paper and electronic patient records, it was possible to assess whether ACP's were a) being created and documented clearly, b) being communicated back to the patient's General Practitioner and c) if the ACP was being transferred onto the patient's Summary Care Record (SCR).

Results On admission to the hospice, 14 out of 26 patients (54\%) had no documented ACP in their notes. Of patients with no ACP, $71 \%$ were known to Community Palliative Care teams. In patients with documented ACPs, $75 \%$ of these plans had been appropriately been communicated to the patient's GP, however $78 \%$ of patients with communication to the GP had no Advance Care Planning details on their SCR.

Conclusions These findings demonstrate the imperative for improved documentation of ACPs in Secondary and Community care and for Summary Care Records to be updated to reflect discussions with patients. Further work will focus on introduction of escalation plans to improve documentation and communication with GP's as well as communication of results, education of professionals involved with Advance Care Planning and re-audit.

\section{INPATIENT ASSESSMENT AND MANAGEMENT OF CONSTIPATION - ARE WE GETTING IT RIGHT?}

Rowena Eason, Angela Halley. Royal Marsden Hospital

\subsection{6/bmjspcare-2019-ASP.146}

Introduction Constipation is a common condition that affects people in all age groups, with complications that can cause significant physical and psychological distress. Validated assessment tools and gold standards for management of constipation are not available due to the lack of robust clinical trials. We conducted an audit to compare local practice of constipation assessment and management to current best practise, aiming to identify areas for improvement and to raise awareness of the significant problem of constipation in inpatients. Our 8 standards were derived from accepted Palliative Care Guidelines and focused on history of constipation, daily bowel assessment and prescription of laxatives.

Methods Data were collected retrospectively from 28 inpatients on general Oncology wards from medical and nursing notes, and medication and bedside charts, in Summer 2018.

Results Unfortunately, only 1 of the 8 standards for good practise was met. Generally, management of constipation and prescription of laxatives was adequate, especially with concurrent use of opioid medication. However, documentation from medical and nursing staff of both assessment and initial and ongoing management was inconsistent and incomplete, and often assumed a level of knowledge of the patient's condition on the part of the reader. The reasons behind these results are likely multi-factorial but may include a lack of confidence in assessing bowels and treating constipation and a lack of understanding of its importance.

Recommendations Following the presentation of this audit we recommended the inclusion of dedicated constipation teaching in the Junior Doctor teaching timetable, with signposting to relevant guidelines, and a review into the use of bowel charts on the ward, to encourage daily use. We are also now in the process of updating the constipation section of our local Symptom Control Guidelines in line with current Palliative Care guidelines. 